\title{
Corrigendum: Noncontextuality with marginal selectivity in reconstructing mental architectures
}

\author{
Ru Zhang and Ehtibar N. Dzhafarov * \\ Department of Psychological Sciences, Purdue University, West Lafayette, IN, USA
}

Keywords: interaction contrast, mental architectures, response time, series-parallel networks, corrigendum

\section{A corrigendum on}

Noncontextuality with marginal selectivity in reconstructing mental architectures by Zhang, R., and Dzhafarov, E. N. (2015). Front. Psychol. 6:735. doi: 10.3389/fpsyg.2015.00735

This corrigendum note points out and corrects two mistakes found in the paper cited in the title. These mistakes do not affect the correctness of the statements proved and expressions derived.

1. In Zhang and Dzhafarov (2015), Lemma 2.6 (p. 7) and Lemma 3.3 (p. 10) are formulated for series-parallel (SP) architectures in which the minimum $(\wedge)$ and maximum $(\vee)$ operations may be intermixed. The proofs are shown for the min-parallel arrangement of the selectively influenced processes, with the correct statement that the max-parallel arrangement is dealt with analogously. However, by an oversight, the proof for the min-parallel arrangement is shown only for homogeneous $\mathrm{SP}_{\wedge}$ architectures, those that cannot contain $\vee$ operations. The statements of the lemmas are correct despite this oversight, because the proofs remain valid if the rightmost $\wedge Y$ and $\wedge X$ in all expressions of the form

$$
\left(\mathrm{SP}^{1}(\ldots) \wedge \mathrm{SP}^{2}(\ldots)+X\right) \underset{\uparrow}{\wedge} Y \text { and }\left(\mathrm{SP}^{1}(\ldots) \wedge \mathrm{SP}^{2}(\ldots) \underset{\uparrow}{X}\right)+Y
$$

are replaced with $\vee Y$ and $\vee X$, respectively.

2. Equations (61) and (62) on p. 9 should be disregarded: one of them, (62), contains typos, and both are shown in the wrong place. These transformations are only valid in the context of Theorem 3.5, for sequential architectures, and this is the only place where they are used, in Equations (63) and (64) on p. 11.

a section of the journal

Frontiers in Psychology

Received: 30 January 2016

Accepted: 11 March 2016

Published: 30 March 2016

Citation:

Zhang $R$ and Dzhafarov EN (2016) Corrigendum: Noncontextuality with marginal selectivity in reconstructing

mental architectures.

Front. Psychol. 7:437.

doi: 10.3389/fpsyg.2016.00437

\section{AUTHOR CONTRIBUTIONS}

All authors listed, have made substantial, direct and intellectual contribution to the work, and approved it for publication.

\section{ACKNOWLEDGMENTS}

This work is supported by NSF grant SES-1155956 and AFOSR grant FA9550-14-1-0318. 


\section{REFERENCES}

Zhang, R., and Dzhafarov, E. N. (2015). Noncontextuality with marginal selectivity in reconstructing mental architectures. Front. Psychol. 6:735. doi: 10.3389/fpsyg.2015.00735

Conflict of Interest Statement: The authors declare that the research was conducted in the absence of any commercial or financial relationships that could be construed as a potential conflict of interest.
Copyright (c) 2016 Zhang and Dzhafarov. This is an open-access article distributed under the terms of the Creative Commons Attribution License (CC BY). The use, distribution or reproduction in other forums is permitted, provided the original author(s) or licensor are credited and that the original publication in this journal is cited, in accordance with accepted academic practice. No use, distribution or reproduction is permitted which does not comply with these terms. 